\section{OPEN ACCESS}

Edited by:

Feng Zhang,

University of Minnesota Twin Cities,

United States

Reviewed by:

Yanpeng Wang,

Chinese Academy of Sciences, China

Gunvant B. Patil,

Texas Tech University, United States

*Correspondence:

Shujie Dong

shujie.dong@syngenta.com

Qiudeng Que

qiudeng.que@syngenta.com

Specialty section: This article was submitted to

Genome Editing in Plants,

a section of the journal

Frontiers in Genome Editing

Received: 21 February 2021 Accepted: 14 April 2021 Published: 12 May 2021

Citation:

Dong S, Qin YL, Vakulskas CA, Collingwood MA, Marand $M$,

Rigoulot S, Zhu L, Jiang Y, Gu W, Fan $C$, Mangum A, Chen Z, Yarnall $M$, Zhong $H$, Elumalai S, Shi $L$ and Que $Q$ (2021) Efficient Targeted Mutagenesis

Mediated by CRISPR-Cas $12 a$

Ribonucleoprotein Complexes in Maize. Front. Genome Ed. 3:670529.

doi: 10.3389/fgeed.2021.670529

\title{
Efficient Targeted Mutagenesis Mediated by CRISPR-Cas12a Ribonucleoprotein Complexes in Maize
}

\begin{abstract}
Shujie Dong ${ }^{1 *}$, Yinping Lucy Qin ${ }^{1}$, Christopher A. Vakulskas ${ }^{2}$, Michael A. Collingwood ${ }^{2}$, Mariam Marand ${ }^{1}$, Stephen Rigoulot ${ }^{1}$, Ling Zhu ${ }^{1}$, Yaping Jiang ${ }^{1}$, Weining Gu ${ }^{1}$, Chunyang Fan ${ }^{1}$, Anna Mangum ${ }^{1}$, Zhongying Chen ${ }^{1}$, Michele Yarnall ${ }^{1}$, Heng Zhong ${ }^{1}$, Sivamani Elumalai ${ }^{1}$, Liang Shi ${ }^{1}$ and Qiudeng Que ${ }^{1 *}$
\end{abstract}

${ }^{1}$ Syngenta Crop Protection, Research Triangle Park, Durham, NC, United States, ${ }^{2}$ Integrated DNA Technologies, Coralville, IA, United States

Recent advances in the development of CRISPR-Cas genome editing technologies have made it possible to perform targeted mutagenesis and precise gene replacement in crop plants. CRISPR-Cas9 and CRISPR-Cas12a are two main types of widely used genome editing systems. However, when CRISPR-Cas12a editing machinery is expressed from a transgene, some chromosomal targets encountered low editing frequency in important crops like maize and soybean. Here, we report efficient methods to directly generate genome edited lines by delivering Cas12a-gRNA ribonucleoprotein complex (RNP) to immature maize embryos through particle bombardment in an elite maize variety. Genome edited lines were obtained at $\sim 7 \%$ frequency without any selection during regeneration via biolistic delivery of Cas12a RNP into immature embryos. Strikingly, the gene editing rate was increased to $60 \%$ on average and up to $100 \%$ in some experiments when the Cas12a RNP was co-delivered with a PMI selectable marker gene cassette and the induced callus cultures were selected with mannose. We also show that use of higher activity Cas12a mutants resulted in improved editing efficiency in more recalcitrant target sequence. The advances described here provide useful tools for genetic improvement of maize.

Keywords: CRISPR-Cas12a, AsCas12a, LbCas12a, mutant Cas12a, ribonucleoprotein delivery, maize genome editing

\section{INTRODUCTION}

Clustered Regularly Interspaced Short Palindromic Repeats (CRISPR)/CRISPR-associated (Cas)adaptive immune systems are widely distributed in nature to defend bacteria from invasion of phages and other mobile genetic elements, such as plasmids and transposons (Sapranauskas et al., 2011; Hille et al., 2018). CRISPR-Cas systems are classified into two main classes and six types (Bayat et al., 2018). The most widely used CRISPR-Cas systems for genome editing are the Types II and V members of the CRISPR/Cas Class 2 systems, Cas9 and Cas12a (aka Cpf1) effectors, respectively (Tang and $\mathrm{Fu}, 2018$ ). CRISPR/Cas9 system has 2 functioning parts, the Cas 9 endonuclease and a guide RNA (gRNA) comprised of two components, a target specific CRISPR RNA (crRNA) and a trans-activating RNA (tracrRNA) (Jinek et al., 2012; Memi et al., 2018). In 
contrast to Cas9, Cas12a (Cpf1) has several distinct features such as T-rich protospacer-adjacent motif (PAM), a guide crRNA without the need for trans-activating RNA (tracrRNA), creation of sticky ends, and ability to self-process crRNA in addition to the DNA nuclease activity (Zetsche et al., 2015; Bayat et al., 2018). These RNA-guided Cas nucleases (RGNs) scan the genome to search for target DNA sequences complementary to the gRNA and generate a DNA double-strand break at the target sequence if there is a proper protospacer adjacent motif (PAM) present. The resulting chromosomal break is repaired by the host DNA repair machineries, either through nonhomologous end joining (NHEJ) or homology-directed repair (HDR) (Chakrabarti et al., 2019).

CRISPR-Cas systems are flexible, precise, simple to use, and efficient. Both CRISPR-Cas9 and CRISPR-Cas12a nucleases have been used widely as highly sequence-specific tools for efficient genome modification (Murugan et al., 2017). CRISPR-Cas systems also have enormous potential for improving global crop production. Their uses in editing genomes have been expanding rapidly among different crops. CRISPR/Cas9 system has been used for genome editing in Arabidopsis, tobacco, rice, sorghum, maize, wheat, poplar, tomato, soybean, petunia, sweet orange as well as liverwort Malcantia polymorpha (El-Mounadi et al., 2020). Several engineered Cas9 enzymes with altered fidelity or target recognition specificity and Cas9 proteins fused with other types of DNA modification enzymes have also been developed (Sretenovic et al., 2020; Zhang Y. et al., 2020). For example, SpCas9-NGv1, an engineered CRISPR-Cas9 recognizing NG PAM edits has been shown to efficiently edit endogenous target sites with NG PAMs in both rice and Arabidopsis; furthermore, target-specific base editors have been generated by fusing NGv1 nickase to cytidine deaminase (Endo et al., 2019).

CRISPR-Cas12a is an alternative system to CRISPR-Cas9 for genome editing (Zetsche et al., 2015). The functionality of Cas12a systems for genome editing has been reported in many plant species including Arabidopsis, cotton, rice, tobacco, tomato and maize (Endo et al., 2016; Begemann et al., 2017; Tang et al., 2017; Xu et al., 2017; Zhong Z. et al., 2018; Lee et al., 2019; Li et al., 2020). LbCas12a variants, targeting alternative noncanonical PAMs, have also been used to edit plant genome sequences and broadened the range of targetable sequences by Cas12a (Li et al., 2018a). Recently, multiplex gene editing with CRISPR-Cas12a and CRISPR-Cas9 systems has also been achieved by expressing the nuclease and crRNA array from a single Pol II promoter for plant genome editing (Wang et al., 2018). Besides, synthesis-dependent repair of Cas12a-induced double strand DNA breaks enabled targeted gene replacement in rice (Li et al., 2018b). However, the editing efficiency is usually lower with Cas12a than with Cas9 and the efficiency varies considerably among different maize targets (Lee et al., 2019). It is possible that temperature is one of the main factors affecting Cas12a editing efficiency (Malzahn et al., 2019). Recently, a high activity variant of Cas12a with temperature tolerance was also reported to resulting in improved editing efficiency in plants (Schindele and Puchta, 2020).

Other than the activity of CRISPR-Cas system, efficient delivery of the editing machinery into the plant cells is key to genome editing applications in crops. In general, stable transformation of DNA expression vectors encoding the CRISPR-Cas components is achieved using Agrobacterium tumefaciens-mediated delivery or direct delivery method such as particle bombardment and protoplast transformation. However, stable transformation of plants is a long process and generates transgenic plants with CRISPR-Cas expression cassettes integrated into their genomes. The integration of DNA construct encoding for the editing machinery in plant genome and its continuous expression might lead to unwanted modifications at off-target genomic sequences (Murovec et al., 2018). Delivering ribonucleoproteins (RNPs) or RNA into cells without DNA is an alternative method to generate transgene-free targeted genome edits (Woo et al., 2015; Svitashev et al., 2016; Zhang et al., 2016; Andersson et al., 2018; Liang et al., 2018). DNA-free delivery is also preferred in crops that are vegetatively propagated since it is not an option to use crossing to breed out the transgene inserts (Murovec et al., 2018; Que et al., 2019). Genome editing through DNA-free delivery with RNP may also ease up regulatory concerns related to transgenes. Editing through transfection of protoplasts with pre-assembled Cas9 RNP complexes has been demonstrated in many plant species (Woo et al., 2015; Malnoy et al., 2016; Svitashev et al., 2016; Andersson et al., 2018; Liang et al., 2018; Murovec et al., 2018; Sant'Ana et al., 2020). However, due to the technical difficulties and long timeline in regenerating plants from protoplasts, the routine use of protoplasts with RNP delivery for generating of stably edited lines has not been reported in economically important crops including cotton, maize, soybean and wheat. On the other hand, some of these crops have well-established high efficiency biolistic transformation systems and successful generation of heritable edits has been achieved using CRISPR-Cas9 in vitro transcripts or RNPs delivered with biolistic method (Svitashev et al., 2016; Liang et al., 2018).

During our research in applying Cas12a editing system to maize we have also observed low editing efficiency in some editing targets when the Cas12a editing machinery was expressed from integrated transgenes. In order to understand why we observed variable editing efficiencies, we have tested several parameters such as different Cas12a enzymes and delivery methods. Here we report efficient gene editing in maize with Cas12a RNP delivered into leaf protoplasts and immature embryos. When RNP was delivered via particle bombardment, the average editing efficiency in immature embryos is above $60 \%$ using co-selection with the phosphomannose isomerase (PMI) marker gene. A large proportion of mutants generated using this protocol carried biallelic mutations, suggesting efficient cleavage of target sites by Cas12a RNP. The results also indicate that Cas12a RNP-mediated editing has much reduced target dependency. We also show that edited plants can be generated with DNA-free RNP delivery method without any selection in a shorter timeline, albeit at much lower efficiency. We also compared different versions of AsCas12a and LbCas12a genes and showed that higher activity Cas12a mutants resulted in improved editing efficiency at recalcitrant target sequences. 


\section{MATERIALS AND METHODS}

\section{Plant Materials}

Syngenta's proprietary elite maize inbred variety NP2222 was used in all experiments. Stock plant care, maize ear production, immature embryo extraction and transformation with PMI as selectable marker gene was carried out as described before (Zhong H. et al., 2018).

\section{Cas12a and crRNA Reagents Used for Plant Transformation}

Cas12a (aka. Cpf1) enzymes including AsCas12a-WT, AsCas12aV3, AsCas12a-Ultra and LbCas12a-V3 (Supplementary Table 1) (Behlke et al., 2018; Vakulskas et al., 2020; Zhang L. et al., 2020) and crRNA were purchased from or provided by IDT (Integrated DNA Technologies, Inc. USA). Guide or spacer sequence of the crRNAs and their corresponding maize gene target are shown in Supplementary Table 2. The crRNA scaffold used for LbCas12a is based on CRISPR-LbCpf1 system, while the crRNA scaffold used for AsCas12a is based on CRISPR-AsCpf1 system (Zetsche et al., 2015).

\section{CRISPR-Cas12a Expression Vectors and Generation of Transgenic Maize Plants}

Binary transformation vector 12672 contains 2 gene expression cassettes, one for the PMI selectable marker gene driven by the maize Ubiquitin-1 promoter and another for the AmCyan fluorescent protein gene driven by the maize Ubiquitin-1 promoter (Zhong H. et al., 2018). For expression of LbCas12a, the rice codon-optimized coding sequence (Tang et al., 2017) was used with 3 bp changes to remove 2 Bsp119I and one RsrII recognition sites. The Cas $12 \mathrm{a}$ transformation vectors (Supplementary Table 3, Supplementary Figure 1) contain 3 expression cassettes: Cas12a expression cassette driven by sugarcane ubiquitin-4 promoter (prSoUbi4) followed by the NOS terminator, crRNA-ribozymes expression cassette and PMI selectable marker cassette. The $B \times 9$ gene target sequences for the crRNAs of these Cas12a vectors are listed in Supplementary Table 3. Transgenic plants were generated through particle bombardment using isolated immature embryos as targets and PMI as a selectable marker (Wright et al., 2001; Chen et al., 2018; Zhong H. et al., 2018). Briefly, immature embryos were isolated from harvested ears at about 9-11 days after pollination and pre-cultured for 1-3 days on osmoticum media. Pre-cultured embryos were then bombarded with the DNA described above using the BioRad PDS-1000 $\mathrm{He}^{\mathrm{TM}}$ Biolistic particle delivery system. Bombarded embryos were then incubated in callus induction media. For $33^{\circ} \mathrm{C}$ treatment, bombarded embryos were incubated at $33^{\circ} \mathrm{C}$ for 2 days before moving back to regular culture temperature at $28^{\circ} \mathrm{C}$. Induced calli were then moved onto mannose selection media. Mannose resistant calli were transferred to regeneration media to induce shoot formation. Shoots were then sub-cultured onto rooting media. Leaf samples were harvested from rooted plants for Taqman ${ }^{\circledR}$ assays to detect mutations in the target site using a previously described real time quantitative polymerase chain reaction (qPCR) Taqman ${ }^{\circledR}$ method (Ingham et al., 2001; Chen et al., 2018).

\section{RNP (Ribonucleoprotein) Complex Preparation, Particle Bombardment, and Plant Regeneration}

To generate Cas12a-crRNA RNP complexes, $0.3 \mathrm{nmol}$ of Cas12 protein and $0.3 \mathrm{nmol}$ of crRNA were mixed together in a total volume of $11 \mu \mathrm{l}$ and incubated at room temperature for $10 \mathrm{~min}$. For RNP delivery alone, the RNPs were coated onto $0.6 \mu \mathrm{m}$ gold particles (Bio-Rad, USA) as follows: $100 \mu \mathrm{l}$ of gold particles (water suspension of $10 \mathrm{mg} / \mathrm{ml}$ ) and $20 \mu \mathrm{l}$ of glycogen $(20$ $\mathrm{mg} / \mathrm{ml}$ ) were added to the premixed RNPs, mixed gently, and then incubated on ice for $10 \mathrm{~min}$. For co-delivery of RNP with DNA, the RNPs and DNA vector plasmid 12672 were coated onto gold particles as follows: $100 \mu \mathrm{l}$ of gold particles (water suspension of $10 \mathrm{mg} / \mathrm{ml})$ and $20 \mu \mathrm{l}$ of glycogen $(20 \mathrm{mg} / \mathrm{ml})$ were added to the premixed RNPs and DNA vector, mixed gently, and incubated on ice for $10 \mathrm{~min}$. The RNP/DNA coated gold particles were then centrifuged at 8,000 $\mathrm{g}$ for $40 \mathrm{~s}$ and the supernatant was removed. The pellet was resuspended with $30 \mu \mathrm{l}$ of sterile water by brief sonication, and then spread onto a macrocarrier disc (10 $\mu \mathrm{l}$ each) followed by air dry in the laminar flow hood (2-4h). Immature embryos were isolated, pre-cultured and bombarded with RNP complex alone or RNP complex with 12672 plasmid DNA as described above for DNA delivery. For experiments with RNP delivery alone and those that did not go through mannose selection, the embryos were cultured on callus induction medium for 2 weeks before transferring to regeneration medium for shoot regeneration. Shoots were then sub-cultured onto rooting media. For RNP-DNA delivery, induced calli were selected on mannose containing media and the resistant calli were placed on regeneration media for transgenic plant production as described above for generation of transgenic plants containing Cas12a expression vectors.

\section{Etiolated Maize Leaf Protoplast Isolation and Transfection}

Protoplasts were isolated from etiolated maize leaves grown under dark conditions as described (Sheen, 1991). Cas12a RNP was assembled as above. Protoplast transfection was carried out as described, with modification (Sant'Ana et al., 2020). Transfection reactions consisted of $5 \times 10^{5}$ protoplasts per reaction and were incubated with PEG solution (40\% PEG4000, 0.6 M Mannitol, $100 \mathrm{mM} \mathrm{CaCl}_{2}$ ) for $15 \mathrm{~min}$. Following termination by W5 solution $(154 \mathrm{mM} \mathrm{NaCl}, 125 \mathrm{mM} \mathrm{CaCl} 2$, $5 \mathrm{mM} \mathrm{KCl}$ and $2 \mathrm{mM} \mathrm{MES,} \mathrm{pH} \mathrm{5.7),} \mathrm{transfected} \mathrm{protoplasts}$ were resuspended in $300 \mu \mathrm{lW} 1$ solution ( $0.6 \mathrm{M}$ Mannitol, $4 \mathrm{mM}$ MES, pH 5.7, $4 \mathrm{mM} \mathrm{KCl),} \mathrm{transferred} \mathrm{to} \mathrm{96-well} \mathrm{clear} \mathrm{bottom}$ microplate and incubated for 2 days in the dark at $28^{\circ} \mathrm{C}$ without shaking. DNA was isolated from transfected protoplasts after 2 days and analyzed for mutant identification (Chen et al., 2018). 


\section{Molecular Analysis and Mutant Identification}

For detection of mutation in immature embryos bombarded with Cas12a-crRNA RNP complex, genomic DNA was extracted from embryos cultured for 2 days after bombardment. Genomic sequences flanking the crRNA target sites were PCR-amplified by specific primers: Bx9-Forward (5' - AAACA CTAAA CACTC CCCTC TG-3'), Bx9-Reverse (5'- GTTTA CCCAT CTCTT TTAAC ACTAT-3'), MIR604-Forward (5'- GGATA TGACT CCACT GACCA - $3^{\prime}$ ) and MIR604-Reverse (5' - CATTT CTCCA TAGCC CGTTT- $3^{\prime}$ ). Amplicons were purified through Ampure $\mathrm{XP}$ beads (Beckman Coulter Inc., Brea, CA) following the manufacturer's instructions. Samples containing $40 \mu \mathrm{l}$ of $>1$ $\mathrm{ng} / \mu \mathrm{l}$ of purified amplicon DNA were sequenced by SeqWell Inc. (Beverly, MA). NGS raw FASTQ files were aligned to corresponding reference sequences. Reads with Indels were extracted. The reads were further filtered by two criteria: first, the variant region must overlap with gRNA target sequence and second, the variant size must be equal to or larger than $2 \mathrm{bp}$. The percentage of reads with edits was calculated using the number of filtered Indels divided by total read count covering the gRNA target region. For analysis of editing in regenerated plants, leaf samples were harvested, and total genomic DNA was extracted from rooted plants for Taqman assays were used to detect putative mutations in target sites (Ingham et al., 2001, Chen et al., 2018). Identified putative mutants were further characterized by Sanger or NGS sequencing analysis.

\section{RESULTS AND DISCUSSION}

\section{Editing of Chromosomal Target Sequences by Cas12a RNP Delivered Into Maize Immature Embryos and Leaf Protoplasts}

DNA-free delivery of editing reagents in the form of protein, mRNA or RNP has great potential to address potential regulatory requirements and public concerns associated with the incorporation of recombinant DNA molecule into edited plants. Successful genome editing using CRISPR-Cas9 RNPs delivered using the biolistic method has been previously demonstrated in wheat and maize using immature embryos as explants (Svitashev et al., 2016, Liang et al., 2018). However, biolistic delivery for Cas12a RNP has yet to be established in maize. We were therefore interested in investigating whether Cas12a RNP could be delivered similarly using particle bombardment to achieve efficient editing in maize. We chose two genomic regions as targets: one is the genic region of $B x 9$ gene which encodes for the UDP-glucosyltransferase involved in benzoxazinoid DIMBOA biosynthesis (von Rad et al., 2001) (GRMZM2G161335 in B73 RefGen_v3) (Sequence File 1 in Supplementary Material) and another is the non-genic region corresponding to the transgene insertion locus of a commercial event MIR604 for root worm control (Chen et al., 2018), (MIR604FS, Sequence File 2 in Supplementary Material).

Table 1 shows the transient editing results of target sequences by AsCas12a-Ultra RNP complexes delivered via particle bombardment. The results show that some genomic targets such as Bx9TS2 can be edited efficiently using AsCas12a RNP. Since only a small percentage of the embryo cells (i.e., those at the scutellum surface and adjacent 1-2 cell layers) can be targeted by microparticles during bombardment, it is expected that a maximum of $5-10 \%$ of the total cells in an immature embryo explant can receive Cas12a RNP. Assuming a 10-day immature maize embryo has around 1,000 cells, it has a maximum of 50100 cells that can be targeted by the delivered RNP complex. Assuming an editing efficiency of $\sim 50 \%$, it is estimated that only $25-50$ cells will get edited and this translates into $2.5-5.0 \%$ of sequence reads having mutant variants. It is remarkable that one of the tested crRNAs (crBx9GS2) resulted in 3.42\% reads with edits, suggesting that AsCas12a RNP has been delivered to many of the surface cells and it is very active in editing the $B \times 9$ gene sequence. Other tested crRNAs resulted in lower editing efficiency, with crMIR604GS2 and crMIR604GS4 having 0.35 and 0.19\% edited reads, and crMIR604GS1 and crMIR604GS3 having the lowest percentage of edited reads $(\sim 0.10 \%)$ (Table 1$)$. The reason for lower editing efficiency is not clear, but it is possible both gRNA and target accessibility may play a role. The results suggest that RNP delivery directly into immature embryos, the transformation target explants, can be a quick way to screen gRNAs for Cas12a-mediated editing.

We also validated the top performing crRNAs (crBx9GS2 and crMIR604GS2) from the immature embryo bombardment assay for editing efficiency using protoplast-mediated transfection (Table 2). AsCas12a-Ultra RNP transfection in maize protoplasts

TABLE 1 | Transient editing of maize chromosomal target sequence by AsCas12a-Ultra RNP complexes delivered via particle bombardment of immature embryos.

\begin{tabular}{|c|c|c|c|c|c|}
\hline Experiment ID & Target site & crRNA & $\begin{array}{c}\text { Total } \\
\text { sequence } \\
\text { read number }\end{array}$ & $\begin{array}{c}\text { Variant } \\
\text { (InDel) read } \\
\text { number }\end{array}$ & $\begin{array}{c}\% \text { Reads with edits } \\
\text { (Mean } \pm \text { SD) } \\
\text { (rep number) }\end{array}$ \\
\hline Blank control 1 & MIR604TS2 & None & 12,841 & 1 & $0.01 \%(n=1)$ \\
\hline Blank control 2 & MIR604TS4 & None & 14,410 & 2 & $0.01 \%(n=1)$ \\
\hline MIR604T1A & MIR604TS1 & crMIR604GS1 & 39,267 & 38 & $0.10 \% \pm 0.10 \%(n=3)$ \\
\hline MIR604T2A & MIR604TS2 & crMIR604GS2 & 37,750 & 133 & $0.35 \% \pm 0.24 \%(n=3)$ \\
\hline MIR604T3A & MIR604TS3 & crMIR604GS3 & 15,922 & 15 & $0.09 \% \pm 0.04 \%(n=3)$ \\
\hline MIR604T4A & MIR604TS4 & crMIR604GS4 & 36,445 & 69 & $0.19 \% \pm 0.18 \%(n=3)$ \\
\hline Bх9T2A & Bx9TS2 & crBx9GS2 & 35,040 & 1,198 & $3.42 \% \pm 0.18 \%(n=3)$ \\
\hline
\end{tabular}


TABLE 2 | Transient editing of maize chromosomal target sequence by AsCas12a-Ultra RNP complexes delivered via PEG-mediated protoplast transfection.

\begin{tabular}{|c|c|c|c|c|c|}
\hline Experiment ID & Target site & crRNA & $\begin{array}{c}\text { Total read } \\
\text { number }\end{array}$ & $\begin{array}{l}\text { Variant } \\
\text { (InDel) read } \\
\text { number }\end{array}$ & $\begin{array}{l}\% \text { Reads with edits } \\
\text { (Mean } \pm \text { SD) } \\
\text { (rep number) }\end{array}$ \\
\hline Blank control 1 & MIR604TS2 & None & 29,788 & 87 & $0.29 \% \pm 0.10 \%(n=3)$ \\
\hline MIR604T2A & MIR604TS2 & crMIR604GS2 & 43,716 & 15,729 & $35.98 \% \pm 5.57 \%(n=6)$ \\
\hline Blank control 2 & Bx9TS2 & None & 71,716 & 153 & $0.21 \% \pm 0.04 \%(n=3)$ \\
\hline Bх9T2A & Bx9TS2 & crBx9GS2 & 149,313 & 30,528 & $20.45 \% \pm 15.06 \%(n=6)$ \\
\hline
\end{tabular}

resulted in high efficiency editing at both targets, with 20.45 and $35.98 \%$ of the reads having edits, respectively. It is surprising that crMIR604GS2-RNP resulted in a high percentage of edited reads in leaf protoplasts, at even higher percentage than was observed for crBx9GS2-RNP (Table 2). It is not clear why the crMIR604GS2-RNP has a higher editing rate in leaf protoplasts as compared to immature embryos. One possible explanation is that the presence of a more open chromatin structure for the MIR604TS2 target locus in leaf vs. immature embryos. It should be noted that protoplast transfection has been widely applied to assess Cas9 RNP activity against different targets in various plant species including maize (Woo et al., 2015; Malnoy et al., 2016; Svitashev et al., 2016; Andersson et al., 2018; Liang et al., 2018; Lin et al., 2018; Murovec et al., 2018; Sant'Ana et al., 2020). Since a much higher percentage of cells can receive RNP in comparison with direct embryo bombardment, protoplast transfection is expected to result (on average) in much higher editing efficiencies. With our maize leaf protoplast transfection experiments with vector expressing green fluorescent protein as reporter, a typical transfection efficiency of $60-70 \%$ is obtained (results not shown). However, protoplast isolation is time-consuming and tedious, and direct bombardment of immature embryos coupled with targeted deep sequencing can offer a straightforward alternative to evaluate gRNA performance directly in the transformation target tissues.

\section{High Efficiency Recovery of Edited Lines by Co-delivery of Cas12a RNP With a Selectable Marker Gene Vector}

Since reasonable editing efficiencies were achieved using AsCas12a RNP in direct immature embryo bombardment, we also tested whether stably edited lines can be recovered from immature embryos bombarded with RNPs targeting $B \times 9$ gene. We co-delivered RNP with a plasmid named pBSC12672 which contains the PMI selectable marker gene (Zhong $\mathrm{H}$. et al., 2018). We tested three different versions of AsCas12a nuclease: AsCas12a-WT, AsCas12a-V3 and AsCas12a-Ultra; the AsCas12a-WT and AsCas12a-V3 proteins contain the wildtype AsCas12a sequence with AsCas12a-V3 also containing an optimized NLS sequence, whereas the AsCas12a-Ultra is an engineered-version of AsCas12a-V3 with mutations that were isolated using directed evolution in bacteria to achieve higher editing efficiencies in living cells (Zhang L. et al., 2020) (Supplementary Table 1).
To optimize mutation rate, we tested multiple parameters including different versions of AsCas12a, helium pressure for bombardment and culture temperature (Supplementary Table 4). Incubation at $37^{\circ} \mathrm{C}$ produced more mutants but transformation efficiency was negatively affected. Overall, the results showed that $1,100 \mathrm{psi}, 0.3 \mathrm{nmol} \mathrm{RNP}$ and culture of bombed embryos at $33^{\circ} \mathrm{C}$ for 2 days immediately after bombardment is a good combination for high efficiency editing of Bx9TS1 target (Supplementary Table 4). We also directly compared performance of AsCas12a-WT with AsCas12aUltra (Table 3). With AsCas12a-WT protein, only about $1.2-7.1 \%$ of transgenic plants derived from immature embryos co-bombarded with RNP and 12672 plasmid DNA showed successful editing at the Bx9TS1 target according to Sanger sequencing results (Table 3 ). The sequencing profile of 5 mutants was examined and these results indicated five distinct deletions had occurred at the expected loci (Supplementary Figure 2). Remarkably, the use of the improved AsCas12a nuclease (AsCas12a-Ultra) resulted in about a 10-fold improvement in editing rate to $68.8 \%$ (Table 3 ). In addition, a high percentage of the edits are biallelic, suggesting high activity of AsCas12a-Ultra in recognizing and cutting the genomic target sequences in maize cells.

\section{Lines With Heritable Edits Can Be Generated Directly From Maize Immature Embryos Bombarded With Cas12a RNP Without Selection}

Considering the high editing rate of AsCas12a RNP in direct immature embryo bombardment (Table 3, Supplementary Table 4), we wondered whether stably edited lines could be recovered from immature embryos that have been bombarded with RNP targeting $B \times 9$ gene in the absence of selection. After AsCas12a RNP was delivered into maize immature embryos, regenerated plant lines (E0) were obtained within 1 month. Putative mutants were identified using high-throughput Taqman assays (Chen et al., 2018) to identify mutations in the Bx9TS2 target sequence (Supplementary Tables 2, 5, Sequence File 1 in Supplementary Material). Mutant plants were further characterized by sequencing to confirm the identity of genomic edits. Table 4 shows that 24 mutants at Bx9TS2 target were identified from a total of 419 immature embryos bombed 
TABLE 3 | Targeted mutagenesis of Bx9 target Bx9TS1 with ribonucleoprotein (RNP) complex of two versions of AsCas12a in maize co-delivered with PMI selectable marker gene vector 12672 .

\begin{tabular}{|c|c|c|c|c|c|c|c|c|}
\hline Treatment & AsCas12a enzyme & $\begin{array}{l}\text { Embryo } \\
\text { explants } \\
\text { used }\end{array}$ & Events ${ }^{\#}$ & $\begin{array}{l}\text { Transformation } \\
\text { frequency }\end{array}$ & $\begin{array}{l}\text { Putative mutant } \\
\text { (Taqman assay) }\end{array}$ & $\begin{array}{l}\text { Biallelic } \\
\text { mutants }\end{array}$ & $\begin{array}{c}\text { Seq. } \\
\text { confirmed } \\
\text { mutants }\end{array}$ & Editing rate* \\
\hline A & AsCas12a-WT & 2,093 & 84 & $4.0 \%$ & 1 & 0 & 1 & $1.2 \%$ \\
\hline$B$ & AsCas12a-WT & 490 & 99 & $20.2 \%$ & 2 & 1 & 2 & $2.0 \%$ \\
\hline C & AsCas12a-WT & 2,165 & 28 & $1.3 \%$ & 2 & 0 & 2 & $7.1 \%$ \\
\hline$D$ & AsCas12a-Ultra & 2,670 & 77 & $2.9 \%$ & 53 & 49 & 53 & $68.8 \%$ \\
\hline
\end{tabular}

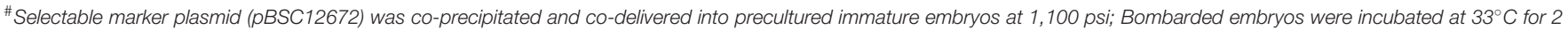
days before moving back to regular culture temperature at $28^{\circ} \mathrm{C}$. Calli selected with mannose.

*Editing rate: number of edited lines/100 regenerated plants.

TABLE 4 | Mutation frequency of Bx9TS2 target in E0 plants directly generated from bombarded immature embryos with RNP without selection.

\begin{tabular}{|c|c|c|c|c|c|c|c|c|}
\hline Treatment & AsCas12a & $\begin{array}{c}\text { Embryo } \\
\text { explants } \\
\text { used }\end{array}$ & $\begin{array}{c}\text { Plants } \\
\text { sampled }^{\&}\end{array}$ & $\begin{array}{c}\text { Biallelic } \\
\text { mutant }\end{array}$ & $\begin{array}{c}\text { Monoallelic } \\
\text { mutant }\end{array}$ & $\begin{array}{l}\text { Total number of } \\
\text { plants with InDel }\end{array}$ & $\begin{array}{l}\text { Editing } \\
\text { rate* }^{*}\end{array}$ & $\begin{array}{c}\text { Editing } \\
\text { efficiency }\end{array}$ \\
\hline A & V3 & 288 & 585 & 11 & 5 & 16 & $2.7 \%$ & $5.6 \%$ \\
\hline B & Ultra & 131 & 204 & 7 & 1 & 8 & $3.9 \%$ & $6.1 \%$ \\
\hline Total & & 419 & 789 & 18 & 6 & 24 & $3.0 \%$ & $5.7 \%$ \\
\hline
\end{tabular}

${ }^{\&}$ For this experiment only, all regenerated shoots from an explant were sampled for mutation analysis. Therefore, some explants had more than 1 shoot.

*Editing rate: number of edited lines/100 regenerated plants.

\#Editing efficiency: number of edited plants/100 starting immature embryo explants.

with AsCas12a RNP and crBx9GS2 gRNA. NGS sequencing confirmed that 16 of the 24 mutants have biallelic mutations.

E0 plants were self-pollinated to produce E1 seeds. Supplementary Table 5 shows that all E1 progeny of the biallelic E0 mutants have biallelic mutations. Of the 3 monoallelic mutant E0 lines, two of them had poor germination, resulting in small number of $\mathrm{E} 1$ lines and thus not meaningful for statistical analysis. However, for unknown reasons line MZKE192601A571A's segregation does not fit the expected 1:2:1 (HOM/HET/WT) ratio. Overall, the results indicated that edits in E0 mutant lines generated using RNP delivery and without selection can be stably inherited to the progeny plants.

\section{Cas12a RNP Results in Comparable or Higher Editing Efficiency in Comparison With Editing Machinery Expressed From Transgenes}

Maize transformation is usually done using particle bombardment or Agrobacterium-mediated delivery. Therefore, genome editing machinery is commonly delivered in the form of DNA expression cassettes. For example, Cas9 nuclease and gRNA are controlled under PolII and PolIII promoters, respectively (Svitashev et al., 2016). We were interested in comparing the relative efficiency of Cas12a editing delivered by either RNP or DNA delivery methods. For biolisticmediated delivery of Cas12a editing machinery targeting Bx9TS1 and Bx9TS2 sites, binary vectors containing various AsCas12a and LbCas12a variants were constructed that express crRNA targeting Bx9TS1 or Bx9TS2 sequence, respectively
(Supplementary Tables 2, 3, Supplementary Figure 1); Each of the vectors contains three expression cassettes: PMI selectable marker, Cas12a and crRNA flanked by self-processing ribozymes (Supplementary Figure 1). Both Cas12a and crRNA were controlled by sugarcane ubiquitin-4 promoter (prSoUbi). Embryos bombarded with different DNA vectors were then selected on mannose to recover transgenic plants. Plants were assayed for editing at Bx9TS1 and Bx9TS2 target sequences.

The results in Table 5 shows that in general the editing rate and efficiency are higher for Bx9TS2 than Bx9TS1 target sites. It is interesting that the editing efficiency of Bx9TS1 is significantly improved when higher activity mutant AsCas12a-Ultra were used in comparison with the wild-type AsCas12a protein (V3). However, the editing efficiency at Bx9TS2 was already very high with the wild-type AsCas12a protein, and the use of improved variant had a negligible effect on editing efficiency (Table 5). We also compared the editing efficiency of two different temperatures on two different editing targets Bx9TS1 and Bx9TS2. Both AsCas12a and LbCas12a vectors worked well for editing of both targets at both 28 and $33^{\circ} \mathrm{C}$ (Table 5). However, higher editing rates at both Bx9TS1 and Bx9TS2 target were achieved when bombed cultures were incubated at $33^{\circ} \mathrm{C}$ (Table 5). For LbCas12a, editing rate at both target sites is somewhat higher with LbCas12a-V3 in comparison with the LbCas12a version with rice optimized codon (Lb, Qi in Table 5).

It is also interesting that the editing rate of Bx9TS1 target sequence with AsCas12a-Ultra RNP was higher than when the editing machinery was expressed from a transgene (Tables 3, 5). The lower editing efficiency with AsCas12a expressed from a transgene is probably due to several reasons. 
TABLE 5 | Editing efficiency of Bx9 target sequences in transgenic plants expressing different versions of Cas12a enzymes.

\begin{tabular}{|c|c|c|c|c|c|c|c|c|c|c|c|}
\hline Vector ID & $\begin{array}{l}\text { Target } \\
\text { site }\end{array}$ & $\begin{array}{l}\text { Cas12a } \\
\text { gene }\end{array}$ & $\begin{array}{l}\text { Culture } \\
\text { temp }\end{array}$ & $\begin{array}{c}\text { Embryo } \\
\text { explants } \\
\text { used }\end{array}$ & $\begin{array}{c}\text { PMI } \\
\text { positive } \\
\text { events+ }\end{array}$ & $\begin{array}{l}\text { Transformation } \\
\text { frequency }\end{array}$ & Biallelic & Monoallelic & $\begin{array}{c}\text { Total } \\
\text { Mutants }\end{array}$ & $\begin{array}{l}\text { Editing } \\
\text { rate }^{\#}\end{array}$ & $\begin{array}{l}\text { Editing } \\
\text { efficiency* }\end{array}$ \\
\hline pBIDT1 & Bx9TS1 & As, V3 & $33^{\circ} \mathrm{C}$ & 714 & 85 & $11.9 \%$ & 2 & 9 & 11 & $12.9 \%$ & $1.5 \%$ \\
\hline pBIDT2 & Bx9TS1 & As, Ultra & $33^{\circ} \mathrm{C}$ & 400 & 34 & $8.5 \%$ & 9 & 5 & 14 & $41.0 \%$ & $3.5 \%$ \\
\hline pBIDT2 & Bx9TS1 & As, Ultra & $28^{\circ} \mathrm{C}$ & 180 & 12 & $6.7 \%$ & 1 & 4 & 5 & $41.0 \%$ & $2.8 \%$ \\
\hline pBIDT3 & Bx9TS1 & Lb, V3 & $33^{\circ} \mathrm{C}$ & 756 & 106 & $14.0 \%$ & 15 & 7 & 22 & $23.6 \%$ & $2.9 \%$ \\
\hline 24096 & Bx9TS1 & Lb, Qi & $33^{\circ} \mathrm{C}$ & 793 & 73 & $17.8 \%$ & 8 & 7 & 13 & $17.8 \%$ & $1.6 \%$ \\
\hline pBIDT4 & Bx9TS2 & As, V3 & $33^{\circ} \mathrm{C}$ & 702 & 132 & $18.8 \%$ & 64 & 35 & 99 & $78.0 \%$ & $14.1 \%$ \\
\hline pBIDT5 & Bx9TS2 & As, Ultra & $33^{\circ} \mathrm{C}$ & 380 & 31 & $8.2 \%$ & 18 & 10 & 28 & $83.9 \%$ & $7.4 \%$ \\
\hline pBIDT5 & Bx9TS2 & As, Ultra & $28^{\circ} \mathrm{C}$ & 205 & 15 & $7.3 \%$ & 3 & 3 & 6 & $33.3 \%$ & $2.9 \%$ \\
\hline pBIDT6 & Bx9TS2 & Lb, V3 & $33^{\circ} \mathrm{C}$ & 720 & 74 & $10.3 \%$ & 30 & 30 & 60 & $83.8 \%$ & $8.3 \%$ \\
\hline 24100 & Bx9TS2 & Lb, Qi & $33^{\circ} \mathrm{C}$ & 787 & 86 & $10.9 \%$ & 30 & 26 & 58 & $67.4 \%$ & $7.4 \%$ \\
\hline
\end{tabular}

\#Editing rate: number of edited lines/100 transgenic events.

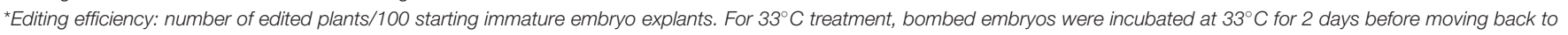
regular culture temperature at $28^{\circ} \mathrm{C}$.

While DNA delivery is efficient, efficient transcription and translation of delivered transgenes can be highly variable depending on the activity of promoters and post-transcriptional regulatory features, and poor codon optimization can also negatively influence overall transgene expression. DNA transgene expression also depends on proper gRNA processing and transport of Cas12a protein back to the correct nuclear compartment for proper RNP assembly; whereas in vitro assembly of Cas12a RNP is very efficient and the preassembled RNP can start doing editing once delivered into the nucleus.

\section{CONCLUSION}

CRISPR-Cas12a is an attractive alternative system to CRISPRCas9 for crop genome editing (Zetsche et al., 2015; Tang et al., 2017). However, it has several limitations in comparison with Cas9 including less flexibility due to its longer PAM sequence requirements (TTTV- $\left.3^{\prime}\right)$, low activity at room temperature, higher percentage of non-functional gRNA (Lee et al., 2019, Malzahn et al., 2019). Therefore, it is highly desirable to have a fast and efficient prescreen system for gRNAs or Cas12a expression vectors to test their functionality before investing resources to carry out stable transformation which is resource-intensive and also usually takes $2-3$ months before regenerated materials are available for molecular analysis. In this report we have demonstrated that the commonly used maize transformation target explants, i.e., immature embryos can be directly bombed with RNPs for assessing their genome editing capability. Alternatively, leaf protoplasts can be an efficient RNP screening system. We have also shown that RNP led to high efficiency editing when delivered into maize immature embryo targets; In addition, stable lines with heritable edits can be efficiently generated via RNP delivery with or without coselection. Finally, we have demonstrated that both AsCas12a, LbCas $12 \mathrm{a}$ and their mutants with enhanced activities can be used to generate targeted genome modifications at high rate. The techniques described in this paper provide us useful tools for precision genome engineering of maize, an important field crop.

\section{DATA AVAILABILITY STATEMENT}

The datasets used and/or analyzed during the current study are available from the corresponding author on reasonable request.

\section{AUTHOR CONTRIBUTIONS}

SD, WG, MY, SR, and QQ conceived and designed experiments. $\mathrm{YQ}, \mathrm{CV}$, and MC constructed the vectors. AM, SE, MM, and SD did transformation experiments. SR did protoplast experiments. CF, YJ, LZ, MY, and WG did assay design and molecular analysis. $\mathrm{CV}$ and MC provided Cas12a enzymes. QQ, HZ, ZC, and LS provided suggestions, research, and laboratory support. SD and QQ wrote the manuscript. All authors contributed to the article and approved the submitted version.

\section{ACKNOWLEDGMENTS}

The authors would like to thank Anna Prairie, Sam Nalapalli, and Xiujuan Su for generating transgenic plants. James Roberts, Rafaela Miranda Lunny Castro, and Tim Strebe for providing maize ears on a timely manner and taking care of the edited plants in greenhouse. David Bradley and Ping Wu for making the media required for tissue culture and transformation. We also like to thank Jianping $\mathrm{Xu}$, Wan Shi, and Ian Jepson for helpful discussions and suggestions.

\section{SUPPLEMENTARY MATERIAL}

The Supplementary Material for this article can be found online at: https://www.frontiersin.org/articles/10.3389/fgeed. 2021.670529/full\#supplementary-material 


\section{REFERENCES}

Andersson, M., Turesson, H., Olsson, N., Fält, A. S., Ohlsson, P., Gonzalez, M. N., et al. (2018). Genome editing in potato via CRISPR-Cas9 ribonucleoprotein delivery. Physiol. Plant 164, 378-384. doi: 10.1111/ppl.12731

Bayat, H., Modarressi, M. H., and Rahimpour, A. (2018). The conspicuity of CRISPR-Cpf1 system as a significant breakthrough in genome editing. Curr. Microbiol. 75, 107-115. doi: 10.1007/s00284-017-1406-8

Begemann, M. B., Gray, B. N., January, E., Gordon, G. C., He, Y., Liu, H., et al. (2017). Precise insertion and guided editing of higher plant genomes using Cpf1 CRISPR nucleases. Sci. Rep. 7:11606. doi: 10.1038/s41598-017-11760-6

Behlke, M. A., Collingwood, M. A., Turk, R., and Vakulskas, C. A. (2018). CRISPR/CPf1 Systems and Methods. United States Patent Application Publication, US20180187176A1. Available online at: https://patents.google. com/patent/US20180187176A1/en (accessed July 5, 2018).

Chakrabarti, A. M., Henser-Brownhill, T., Monserrat, J., Poetsch, A. R., Luscombe, N. M., and Scaffidi, P. (2019). Target-specific precision of CRISPR-mediated genome editing. Mol. Cell. 73, 699-713. doi: 10.1016/j.molcel.2018.11.031

Chen, Z., Kim, M., Chilton, M. D., Zhong, H., Gu, W., Jiang, Y., et al. (2018). Methods and Compositions for Identifying and Enriching for Cells Comprising Site Specific Genomic Modifications. United States Patent 9,963,710.

El-Mounadi, K., Morales-Floriano, M. L., and Garcia-Ruiz, H. (2020). Principles, applications, and biosafety of plant genome editing using CRISPR-Cas9. Front. Plant Sci. 11:56. doi: 10.3389/fpls.2020.00056

Endo, A., Masafumi, M., Kaya, H., and Toki, S. (2016) Efficient targeted mutagenesis of rice and tobacco genomes using Cpf1 from Francisella novicida. Sci. Rep. 6:38169. doi: 10.1038/srep38169

Endo, M., Mikami, M., Endo, A., Kaya, H., Itoh, T., Nishimasu, H., et al. (2019). Genome editing in plants by engineered CRISPR-Cas9 recognizing NG PAM. Nat. Plants 5, 14-17. doi: 10.1038/s41477-018-0321-8

Hille, F., H., Richter,Wong, S. P., Bratovič, M., Ressel, S., and Charpentier, E. (2018). The biology of CRISPR-Cas: backward and forward. Cell 172, 1239-1259. doi: 10.1016/j.cell.2017.11.032

Ingham, D. J., Beer, S., Money, S., and Hansen, G. (2001). Quantitative real-time PCR assay for determining transgene copy number in transformed plants. Biotechniques 31, 132-134. doi: 10.2144/01311rr04

Jinek, M., Chylinski, K., Fonfara, I., Hauer, M., Doudna, J. A., and Charpentier, E. (2012). A programmable dual-RNA-guided DNA endonuclease in adaptive bacterial immunity. Science 337, 816-821. doi: 10.1126/science.1225829

Lee, K., Zhang, Y., Kleinstiver, B. P., Guo, J. A., Aryee, M. J., Miller, J., et al. (2019). Activities and specificities of CRISPR/Cas9 and Cas12a nucleases for targeted mutagenesis in maize. Plant Biotech. J. 17, 362-372. doi: 10.1111/pbi.12982

Li, S., Li, J., Zhang, J., Du, W., Fu, J., Sutar, S., et al. (2018b). Synthesis-dependent repair of Cpf1-induced double strand DNA breaks enables targeted gene replacement in rice. J. Exp. Bot. 69, 4715-4721. doi: 10.1093/jxb/ery245

Li, S., Zhang, X., Wang, W., Guo, X., Wu, Z., Du, W., et al. (2018a). Expanding the scope of CRISPR/Cpf1-mediated genome editing in rice. Mol. Plant 11, 995-998. doi: 10.1016/j.molp.2018.03.009

Li, S., Zhang, Y., Xia, L., and Qi, Y. (2020). CRISPR-Cas12a enables efficient biallelic gene targeting in rice. Plant Biotech. J. 18, 1351-1353. doi: 10.1111/pbi.13295

Liang, Z., Chen, K., Zhang, Y., Liu, J., Yin, K., Qiu, J. L., et al. (2018). Genome editing of bread wheat using biolistic delivery of CRISPR/Cas9 in vitro transcripts or ribonucleoproteins. Nat. Protocol. 13, 413-430. doi: $10.1038 /$ nprot.2017.145

Lin, C. S., Hsu, C. T., Yang, L. H., Lee, L. Y., Fu, J. Y., Cheng, Q. W., et al. (2018). Application of protoplast technology to CRISPR/Cas9 mutagenesis: from single-cell mutation detection to mutant plant regeneration. Plant Biotech. J. 16, 1295-1310. doi: 10.1111/pbi.12870

Malnoy, M., Viola, R., Jung, M. H., Koo, O. J., Kim, S., Kim, J. S., et al. (2016). DNAfree genetically edited grapevine and apple protoplast using CRISPR/Cas9 ribonucleoproteins. Front. Plant Sci. 7:1904. doi: 10.3389/fpls.2016. 01904

Malzahn, A. A., Tang, X., Lee, K., Ren, Q., Sretenovic, S., Zhang, Y., et al. (2019). Application of CRISPR-Cas12a temperature sensitivity for improved genome editing in rice, maize, and Arabidopsis. BMC Biol. 17:9. doi: $10.1186 /$ s12915-019-0629-5
Memi, F., Ntokou, A., and Papangeli, I. (2018). CRISPR/Cas9 gene-editing: research technologies, clinical applications and ethical considerations. Semin. Perinatol. 42, 487-500. doi: 10.1053/j.semperi.2018.09.003

Murovec, J., Guček, K., Bohanec, B., Avbelj, M., and Jerala, R. (2018). DNA-free genome editing of Brassica oleracea and B. rapa protoplasts using CRISPR-Cas9 ribonucleoprotein complexes. Front. Plant Sci. 9:1594. doi: $10.3389 /$ fpls.2018.01594

Murugan, K., Babu, K., Sundaresan, R., Rajan, R., and Sashital, D. G. (2017). The revolution continues: Newly discovered systems expand the CRISPR-Cas toolkit. Mol Cell. 68, 15-25. doi: 10.1016/j.molcel.2017.09.007

Que, Q., Chen, Z., Kelliher, T., Skibbe, D., Dong, S., and Chilton, M. D. (2019). Plant DNA repair pathways and their applications in genome engineering. Methods Mol Biol. 1917, 3-24. doi: 10.1007/978-1-4939-8991-1_1

Sant'Ana, R. R. A., Caprestano, C. A., Nodari, R. O., and AgapitoTenfen, S. Z. (2020). PEG-delivered CRISPR-Cas9 ribonucleoproteins system for gene-editing screening of maize protoplasts. Genes 11:1029. doi: 10.3390/genes11091029

Sapranauskas, R., Gasiunas, G., Fremaux, C., Barrangou, R., Horvath, P., and Siksnys, V. (2011). The Streptococcus thermophilus CRISPR/Cas system provides immunity in Escherichia coli. Nucleic Acids Res. 39, 9275-9282. doi: 10.1093/nar/gkr606

Schindele, P., and Puchta, H. (2020). Engineering CRISPR/LbCas12a for highly efficient, temperature-tolerant plant gene editing. Plant Biotech. J. 18, 1118-1120. doi: 10.1111/pbi.13275

Sheen, J. (1991). Molecular mechanisms underlying the differential expression of maize pyruvate, orthophosphate dikinase genes. Plant Cell 3, 225-245. doi: $10.1105 /$ tpc.3.3.225

Sretenovic, S., Yin, D., Levav, A., Selengut, J. D., Mount, S. M., and Qi, Y. (2020). Expanding plant genome-editing scope by an engineered iSpyMacCas 9 system that targets A-rich PAM sequences. Plant Commun. 2:100101. doi: 10.1016/j.xplc.2020.100101

Svitashev, S., Schwartz, C., Lenderts, B., Young, J., and Cigan, A. M. (2016). Genome editing in maize directed by CRISPR-Cas9 ribonucleoprotein complexes. Nat. Commun. 7:13274. doi: 10.1038/ncomms13274

Tang, X., Lowder, L., Zhang, T., Malzahn, A. A., Zheng, X., and Voytas, D. F. (2017). A CRISPR-Cpf1 system for efficient genome editing and transcriptional repression in plants. Nat. Plants 3:17018. doi: 10.1038/nplants.2017.103

Tang, Y., and Fu, F. (2018). Class 2 CRISPR/Cas: an expanding biotechnology toolbox for and beyond genome editing. Cell Biosci. 8:59. doi: 10.1186/s13578-018-0255-x

Vakulskas, C. A., Bode, N. M., Collingwood, M. A., and Beaudoin, S. (2020). Cas12a Mutant Genes and Polypeptides Encoded by Same. United States Patent Application Publication, US20200216825A1. Available online at: https:// patents.google.com/patent/US20200216825A1/en (accessed July 9, 2020).

von Rad, U., Hüttl, R., Lottspeich, F., Gierl, A., and Frey, M. (2001). Two glucosyltransferases are involved in detoxification of benzoxazinoids in maize. Plant J. 28, 633-642. doi: 10.1046/j.1365-313x.2001.01161.x

Wang, M., Mao, Y., Lu, Y., Wang, Z., Tao, X., and Zhu, J. K. (2018). Multiplex gene editing in rice with simplified CRISPR-Cpf1 and CRISPR-Cas9 systems. J. Integr. Plant Biol. 60, 626-631. doi: 10.1111/jipb.12667

Woo, J. W., Kim, J., Kwon, S. I., Corvalan, C., Cho, S. W., et al. (2015). DNA-free genome editing in plants with preassembled CRISPR-Cas9 ribonucleoproteins. Nat. Biotechnol. 33, 1162-1164. doi: 10.1038/nbt.3389

Wright, M., Dawson, J., Dunder, E., Suttie, J., Reed, J., Kramer, C., et al. (2001). Efficient biolistic transformation of maize (Zea mays L.) and wheat (Triticum aestivum $\mathrm{L}$.) using the phosphomannose isomerase gene, pmi, as the selectable marker. Plant Cell Rep. 20, 429-436. doi: 10.1007/s002990100318

Xu, R., Qin, R., Li, H., Li, D., Li, L., Wei, P., et al. (2017) Generation of targeted mutant rice using a CRISPR-Cpf1 system. Plant Biotech. J. 15, 713-717. doi: $10.1111 / \mathrm{pbi} .12669$

Zetsche, B., Gootenberg, J. S., Abudayyeh, O. O., Slaymaker, I. M., Makarova, K. S., Essletzbichler, P., et al. (2015). Cpf1 is a single RNA-guided endonuclease of a class 2 CRISPR-Cas system. Cell 163, 759-771. doi: 10.1016/j.cell.2015.09.038

Zhang, L., Vakulskas, C. A., Bode, N. M., Collingwood, M. A., Beltz, K. R., and Behlke, M. A. (2020). Novel Mutations That Enhance the DNA Cleavage Activity of Acidaminococcus sp. cpf1. United States Patent Application Publication, US20200109382A1. Available online at: https://patents.google.com/patent/ US20200109382A1/en (accessed April 9, 2020). 
Zhang, Y., Liang, Z., Zong, Y., Wang, Y., Liu, J., and Chen, K. (2016). Efficient and transgene-free genome editing in wheat through transient expression of CRISPR/Cas9 DNA or RNA. Nat. Commun. 7:12617. doi: 10.1038/ncomms12617

Zhang, Y., Pribil, M., Palmgren, M., and Gao, C. (2020). A CRISPR way for accelerating improvement of food crops. Nat. Food 1, 200-205. doi: 10.1038/s43016-020-0051-8

Zhong, H., Elumalai, S., Nalapalli, S., Richbourg, L., Prairie, A., Bradley, D., et al. (2018). Advances in Agrobacterium-mediated maize transformation. Methods Mol. Biol. 1676, 41-59. doi: 10.1007/978-1-4939-7315-6_3

Zhong, Z., Zhang, Y., You, Q., Tang, X., Ren, Q., Liu, S., et al. (2018). Plant genome editing using FnCpf1 and LbCpf1 nucleases at redefined and altered PAM sites. Mol. Plant 11, 999-1002. doi: 10.1016/j.molp.2018.03.008
Conflict of Interest: SD, YQ, MM, SR, LZ, YJ, WG, CF, AM, ZC, MY, HZ, SE, LS, and QQ were employed by Syngenta Crop Protection, LLC. CV and $\mathrm{MC}$ are employed by Integrated DNA Technologies which manufactures genome editing reagents.

Copyright (C 2021 Dong, Qin, Vakulskas, Collingwood, Marand, Rigoulot, Zhu, Jiang, Gu, Fan, Mangum, Chen, Yarnall, Zhong, Elumalai, Shi and Que. This is an openaccess article distributed under the terms of the Creative Commons Attribution License (CC BY). The use, distribution or reproduction in other forums is permitted, provided the original author(s) and the copyright owner(s) are credited and that the original publication in this journal is cited, in accordance with accepted academic practice. No use, distribution or reproduction is permitted which does not comply with these terms. 